\title{
COMPARING CORONAL AND HELIOSPHERIC MAGNETIC FIELDS OVER SEVERAL SOLAR CYCLES
}

\author{
J. S. Koskela ${ }^{1}$, I. I. VirTanen ${ }^{1}$, and K. Mursula ${ }^{1}$ \\ University of Oulu, P.O. Box 3000, FI-90014 Oulu, Finland; jennimari.koskela@oulu.fi \\ Received 2016 August 18; revised 2016 November 10; accepted 2016 November 15; published 2017 January 18
}

\begin{abstract}
Here we use the PFSS model and photospheric data from Wilcox Solar Observatory, SOHO/MDI, SDO/HMI, and SOLIS to compare the coronal field with heliospheric magnetic field measured at 1 au, compiled in the NASA/ NSSDC OMNI 2 data set. We calculate their mutual polarity match and the power of the radial decay, $p$, of the radial field using different source surface distances and different number of harmonic multipoles. We find the average polarity match of $82 \%$ for the declining phase, $78 \%-79 \%$ for maxima, $76 \%-78 \%$ for the ascending phase, and $74 \%-76 \%$ for minima. On an average, the source surface of $3.25 R_{S}$ gives the best polarity match. We also find strong evidence for solar cycle variation of the optimal source surface distance, with highest values $\left(3.3 R_{S}\right)$ during solar minima and lowest values $\left(2.6 R_{S}-2.7 R_{S}\right)$ during the other three solar cycle phases. Raising the number of harmonic terms beyond 2 rarely improves the polarity match, showing that the structure of the HMF at 1 au is most of the time rather simple. All four data sets yield fairly similar polarity matches. Thus, polarity comparison is not affected by photospheric field scaling, unlike comparisons of the field intensity.
\end{abstract}

Key words: Sun: corona - Sun: magnetic fields - Sun: photosphere

\section{INTRODUCTION}

The connection between the solar and the heliospheric magnetic fields has been an intriguing problem since the IMP 1 satellite first discovered the sector structure in the heliosphere (Ness \& Wilcox 1964). The heliospheric magnetic field (HMF) is frozen in the solar wind plasma which, at its origins, is affected by the coronal magnetic field. The coronal magnetic field is difficult to measure, but the potential field source surface (PFSS) model (Altschuler \& Newkirk 1969; Schatten et al. 1969) has long been used to estimate the coronal field from the measured photospheric field. The PFSS model is simpler than magnetohydrodynamic (MHD) models, but the large-scale configuration of the field calculated with the PFSS model is as good as the one calculated with MHD models (Wiegelmann et al. 2015). However, the radial component of the magnetic field in the PFSS model shows latitude dependence. The field is zero close to the neutral line, the coronal projection of the heliospheric current sheet (HCS), and grows toward higher latitudes. This is a weakness in the model, since the Ulysses measurements have shown that there is no latitude dependence (Smith \& Balogh 1995).

In the PFSS model the coronal field is calculated up to the source surface distance $\left(r_{s s}\right)$, where the magnetic field lines are assumed to be radial. The classic source surface distance of $2.5 R_{S}\left(R_{S}\right.$ is the radius of the Sun) was introduced by Hoeksema et al. (1983). However, some studies (Lee et al. 2011; Arden et al. 2014) suggest that the source surface distance should be treated as a free parameter.

In this paper we use the PFSS model and photospheric magnetic field measurements to calculate the coronal magnetic field and to compare it with the heliospheric magnetic field measured at the Earth's orbit. We study the effect of varying the source surface distance and the number of multipoles in the spherical harmonic expansion on the coronal field and its prediction of the HMF at 1 au. We also use several data sets (WSO, SOLIS, MDI, HMI) of photospheric field observations

\footnotetext{
${ }^{1}$ ReSoLVE Centre of Excellence, Space Climate Research Unit, University of Oulu.
}

and compare their predictions. The paper is organized as follows: In Section 2 we introduce the data sources used in the analysis and discuss our methods. In Section 3 we compare the modeled and observed HMF polarities, and in Section 4 the magnitudes of the radial component. In Section 5 we discuss our results, and we give our summary in Section 6 .

\section{DATA AND METHODS}

We use photospheric data primarily from WSO (Wilcox Solar Observatory), but also from SOLIS (Synoptic Optical Long-Term Investigations of the Sun), MDI/SOHO (Michelson Doppler Imager/Solar and Heliospheric Observatory), and HMI/SDO (Helioseismic and Magnetic Imager/Solar Dynamics Observatory). Table 1 lists the resolutions of the synoptic maps and the times and Carrington rotations included in the analysis. The way the synoptic maps are constructed from single measurements may have a small effect on the resulting coronal field. Another uncertainty is caused by the different coverage of solar polar regions by the observatories and the different methods in filling them. In this paper the synoptic maps have been preprocessed in the same way as in Virtanen \& Mursula (2016).

The heliospheric parameters (solar wind and HMF) are retrieved from the NASA/NSSDC OMNI 2 data set. We use the hourly OMNI 2 data, which we smooth with a $25 \mathrm{hr}$ running mean in order to remove daily variations. We require at least $7 \mathrm{hr}$ of data for each running mean window, otherwise the hour is neglected. According to Lockwood et al. (2006), the time period should be chosen so that the small-scale structures originating during heliospheric propagation are averaged out but the large-scale, actual sector structure is not. Approximately 1 day averaging period was found to be a good compromise. The window of $25 \mathrm{hr}$ corresponds to 13.7 in Carrington longitude.

The main assumption of the PFSS model is that there are no currents between the photosphere and the source surface. The magnetic field is assumed to be radial in the photosphere and on the source surface. Field lines that pass the source surface are open outside the source surface and contribute to the HMF. 
Table 1

List of Magnetographs Used in this Study (First Column), Their Latitudinal $\left(N_{\theta}\right)$ and Longitudinal $\left(N_{\phi}\right)$ Resolution (Second Column), the Carrington Rotations Used for this Study (Third Column), and the Dates Corresponding to These Rotations (Fourth Column)

\begin{tabular}{lccc}
\hline \hline Observatory & Resolution $\left(N_{\theta} \times N_{\phi}\right)$ & Rotations & Data period \\
\hline WSO & $73 \times 30$ & $1643-2177$ & 1976 Jun 23-2016 Jun 6 \\
MDI & $3600 \times 1080$ & $1912-2102$ & 1996 Jul 25-2010 Oct 30 \\
HMI & $3600 \times 1440$ & $2097-2177$ & 2010 May 19-2016 Jun 6 \\
SOLIS & $360 \times 180$ & $2008-2177$ & 2003 Sep 26-2016 Jun 6 \\
\hline
\end{tabular}

The amount of open magnetic flux in the heliosphere depends on the source surface distance. If the source surface distance is small, more field lines will cross the source surface and the HMF will be stronger. On the other hand, if the source surface distance is large, more field lines will close within the source surface distance and the HMF will be weaker.

Mathematically, the assumption of no electric currents between the photosphere and source surface leads to the Laplace equation, which can be solved with spherical harmonics. The radial magnetic field (at distance $r$ from the center of the Sun, co-latitude $\theta$ and longitude $\phi$ ) then becomes (Altschuler \& Newkirk 1969; Sun 2009)

$$
\begin{aligned}
B_{r}(r, \theta, \phi)= & \sum_{n=1}^{n_{\max }} \sum_{m=0}^{n} P_{n}^{m}(\cos \theta)\left(g_{n m} \cos m \phi+h_{n m} \sin m \phi\right) \\
& \times\left(\frac{R_{S}}{r}\right)^{n+2} \frac{n+1+n\left(r / r_{s S}\right)^{2 n+1}}{n+1+n\left(R_{S} / r_{s S}\right)^{2 n+1}},
\end{aligned}
$$

where $P_{n}^{m}(\cos \theta)$ are the Legendre polynomials, $n_{\max }$ is the order of harmonic expansion, and $g_{n m}$ and $h_{n m}$ are the harmonic coefficients that are obtained from the photospheric maps as follows:

$$
\begin{aligned}
& g_{n m}=\frac{2 n+1}{N_{\theta} N_{\phi}} \sum_{i=1}^{N_{\theta}} \sum_{j=1}^{N_{\phi}} B_{r}\left(R_{S}, \theta_{i}, \phi_{j}\right) P_{n}^{m}\left(\cos \theta_{i}\right) \cos m \phi_{j} \\
& h_{n m}=\frac{2 n+1}{N_{\theta} N_{\phi}} \sum_{i=1}^{N_{\theta}} \sum_{j=1}^{N_{\phi}} B_{r}\left(R_{S}, \theta_{i}, \phi_{j}\right) P_{n}^{m}\left(\cos \theta_{i}\right) \sin m \phi_{j} .
\end{aligned}
$$

Here $N_{\theta}$ and $N_{\phi}$ are the number of bins of the synoptic map in the latitudinal and longitudinal direction, respectively; $\theta_{i}$ is the co-latitude of the $i$ th bin; and $\phi_{j}$ is the longitude of the $j$ th bin. $B_{r}\left(R_{S}, \theta_{i}, \phi_{j}\right)$ is the measured radial component of the photospheric field in the bin $i, j$. Note that in Equation (1) the nonphysical magnetic monopole term is not taken into account, since the first term in the calculation is $n=1$ (dipole).

The PFSS model field is compared to the heliospheric magnetic field measured at $1 \mathrm{au}$. To do this, the HMF observations need to be traced back to the source surface by calculating the time delay of the solar wind propagation. We assume that solar wind fronts with different velocities do not interact with each other, i.e., that the solar wind speed profile depends only on the speed measured at 1 au. Figure 1 shows the time delay $\Delta t$ (in hours) from the corona to 1 au as a function of solar wind speed measured at 1 au for two different cases: using Parker's solar wind speed profile (Parker 1958), or using a constant speed from corona to $1 \mathrm{au}$. The time delay is calculated by integrating the speed profile from source surface to $1 \mathrm{au}$. For each case, we have calculated the time delay using four source surface distances. Figure 1 also depicts the histogram distribution of solar wind speeds measured at 1 au. As noted previously, the hourly resolution of solar wind speed has been smoothed to $25 \mathrm{hr}$ running means.

Figure 1 shows a clear problem with Parker's model: the time delay grows very large for low solar wind speeds, especially for the smaller source surface distances. The histogram shows that the lowest measured (smoothed) velocities are between 200 and $300 \mathrm{~km} \mathrm{~s}^{-1}$, the minimum speed being $210 \mathrm{~km} \mathrm{~s}^{-1}$. For a solar wind of this speed at $1 \mathrm{au}$ originating from the source surface distance of $2.5 R_{S}$, the time delay according to Parker's model would be almost 2 years, which is unrealistically long. The reason is that in Parker's model, the solar wind acceleration is very slow at small distances. On the other hand, for the constant solar wind speed model, the time delays are more reasonable and, naturally, almost independent of the source surface distance. For example, for the solar wind speed of $450 \mathrm{~km} \mathrm{~s}^{-1}$ and $r_{s s}=1.5 R_{S}$, the delay is 4 days, and for $210 \mathrm{~km} \mathrm{~s}^{-1}$, it is 8 days. For these reasons we use the constant solar wind speed model for tracing the magnetic field observed at 1 au to the source surface.

We have also tested if the coronal mass ejections (CME) have an effect on predicting the intensity and polarity of the HMF. We removed the hours of CMEs in the solar wind by using the classification of solar wind into three main types by Richardson \& Cane (2012). However, this had a very small effect on the success of both intensity and polarity prediction (80\% on average when CMEs are removed, compared to $79 \%$ when they are included). Therefore we do not remove the times of CMEs from the comparisons made in this study.

The procedure is as follows: we first determine the source surface coordinates of the origin of the field line measured at $1 \mathrm{au}$, and then calculate the PFSS solution at that point. The source longitude of the solar wind can be calculated, assuming that the solar wind moves radially outward after leaving the source surface. Then the source longitude is the longitude of the central meridian of the Sun at $t-\Delta t$. The source latitude is chosen to be equal to the Earth's heliographic latitude at the time $t$. We select the Carrington rotation, including the emission time $t-\Delta t$, and calculate the harmonic coefficients with the correct photospheric synoptic map. With the harmonic coefficients we can obtain the PFSS coronal field value at the correct source latitude and longitude, with the chosen source surface distance. Then the coronal field and the corresponding heliospheric field at 1 au can be compared.

To account for the $25 \mathrm{hr}$ smoothing in OMNI 2 data, for each hour at $1 \mathrm{au}$, we calculated the PFSS solution at 25 points with a longitudinal separation of 0.55 (corresponding to $1 \mathrm{hr}$ ), i.e., with longitudes ranging from $\lambda_{0}-12 \times 0.55$ to $\lambda_{0}+12 \times$ 0.55 . These 25 values were then averaged. 

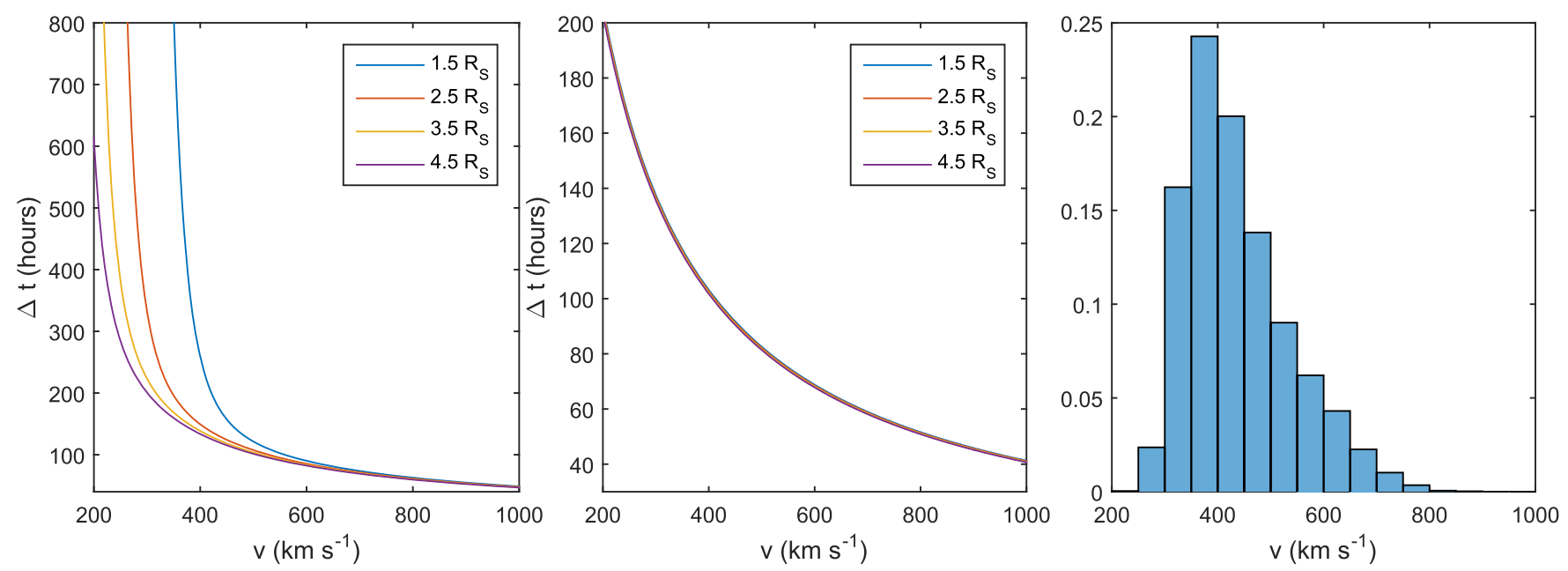

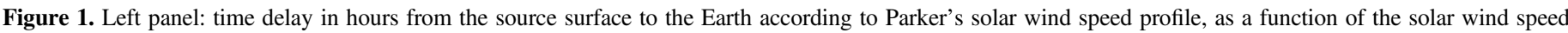

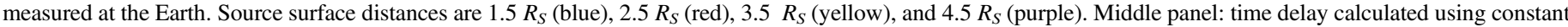

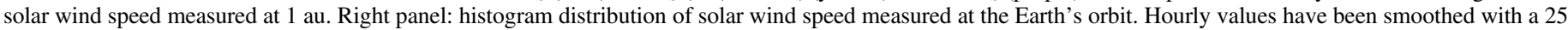
hr running mean.

\section{HMF POLARITY MATCH}

\subsection{Dependence on Source Surface Distance}

We have calculated the polarity match percentage for each Carrington rotation by comparing the signs of the radial component of the magnetic field measured at 1 au and the coronal field. Only rotations which had more than 200 hourly data points in OMNI 2 data (i.e., data coverage of more than $30 \%$ ) were included. We have also calculated a 13-rotation running mean (requiring a minimum of four rotations for each 13-rotation window) to get rid of a possible yearly variation that might result (e.g., from the vantage point effect; Virtanen \& Mursula 2010).

Figure 2 shows the polarity match for WSO photospheric data with $n_{\max }=9$ and for several source surface distances: $1.5 R_{S}, 2.5 R_{S}, 3.5 R_{S}$, and $4.5 R_{S}$. The smallest source surface distance gives by far the worst match at almost all times, never yielding a better match than one of the larger source surface distances. The average polarity match over the whole data series for the source surface distance of $1.5 R_{S}$ is $69.48 \%$. On the other hand, the percentages of the three larger source surface distances are typically very similar, with mutual differences remaining very small. The average polarity match calculated over the whole period is only slightly better for $r_{s s}=3.5 R_{S}(78.59 \%)$ than for $2.5 R_{S}(78.23 \%)$ or 4.5 $R_{S}(77.91 \%)$.

We have further studied the variation of the overall polarity match for a larger range of source surface distances from $1.25 R_{S}$ to $8.5 R_{S}$, with a step of $0.25 R_{S}$. Figure 3 shows that the highest polarity match of $78.6 \%$ is reached with the source surface distance of $3.25 R_{S}$. At the source surface distance of approximately $2.25 R_{S}$, the polarity match reaches the level within two percentage points from the highest value, and after $5.75 R_{S}$ decreases below it. Note also that the decline of polarity match beyond the optimal distance is very slow, and remains above $70 \%$ even for the source surface distance of $8.5 R_{S}$.

Figure 2 shows large temporal variations in the polarity match percentage. The match seems to have at least one maximum during the declining phase of every solar cycle. (The bottom panel of Figure 2 shows the 13 month smoothed monthly sunspot number from 1976 to 2015 as a reference.) To assess this in a more quantitative way, Figure 4 shows the average polarity match percentage for each of the four phases of the solar cycle. We use the recently suggested solar cycle phase function (Maliniemi et al. 2014), which divides the solar cycle into four phases according to the sunspot minimum and maximum times (ftp://ftp.ngdc. noaa.gov/STP/space-weather/solar-data/solar-indices/sunspotnumbers/cycle-data/table_cycle-dates_maximum-minimum.txt; maximum of cycle 24 is taken to be in 2014 April; extended here until 2015 December). In the solar cycle phase function the sunspot minimum is defined to be $0^{\circ}$ and the sunspot maximum $180^{\circ}$. The ascending phase and the declining phase are located between the minimum and maximum phases so that each phase of the solar cycle is $90^{\circ}$ long.

Figure 4 shows that during the declining phase, the polarity match percentages are clearly higher for all $r_{s s}$ values than during other cycle phases. The highest percentages in the declining phase, approximately $82 \%$, are found for the two source surface distances of $2.5 R_{S}$ and $3.5 R_{S}$. During the other three solar cycle phases the average match percentages for the three largest source surface distances are approximately $78 \%$ $79 \%$ during maximum, $76 \%-78 \%$ during the ascending phase, and $74 \%-76 \%$ during minimum. The smallest source surface distance gives a significantly lower polarity match than the three larger source surface distances during all four solar cycle phases, but the difference is drastic during the minimum phase.

Using the previously mentioned 30 source surface distances $\left(1.25 R_{S}-8.5 R_{S}\right)$ and the related match percentages, we have determined the optimal source surface distance for each Carrington rotation in the following way. Note first that despite the overall decrease of match percentages with increasing source surface distance beyond $r_{s s}=3.25 R_{S}$ (see Figure 3), the individual rotations may have very similar percentages for several $r_{s s}$ values, some even fairly far from each other. Therefore we have selected the optimal source surface distance as the smallest $r_{s s}$ value which is at most two percentage points lower than the highest value. The 13-rotation running means of 

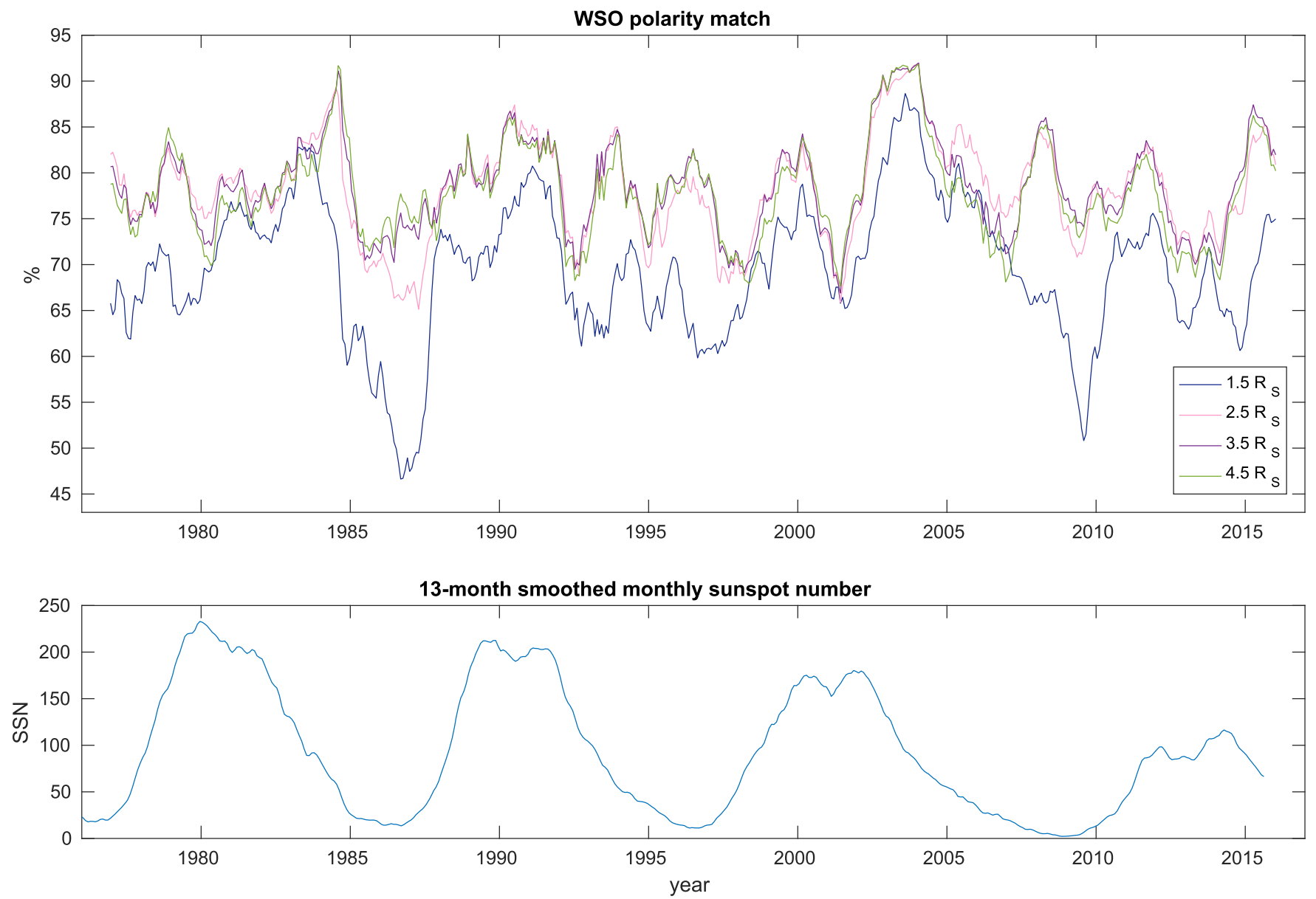

Figure 2. Top panel: the 13-rotation running means of the rotational polarity match percentages $\left(n_{\max }=9\right)$ for four different source surface distances: $1.5 R_{S}$ (blue), $2.5 R_{S}$ (pink), $3.5 R_{S}$ (purple), and $4.5 R_{S}$ (green). Bottom panel: the 13 month smoothed monthly sunspot number (Source: WDC-SILSO, Royal Observatory of Belgium, Brussels).

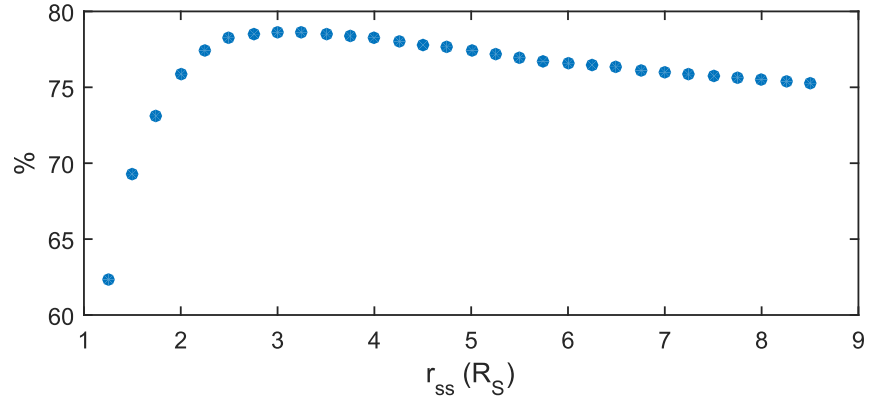

Figure 3. Average polarity match percentage over the whole WSO data period, using source surface distances from $1.25 R_{S}$ to $8.5 R_{S}$, with a step of $0.25 R_{S}$.

the optimal source surface distances are given in the upper panel of Figure 5. The largest optimal source surface distance of about $4.4 R_{S}$ was reached in 1985 and 1986, during the minimum of solar cycle 21 . The optimal source surface distance was also large in 1997, during the minimum of cycle 22 , and in 2009 , the minimum of cycle 23 . Other peaks in the optimal source surface distance were found during the declining phase of all studied cycles (1982-1983, 1992-1993, $1995,2003,2015)$, and in 1978, during the ascending phase of cycle 21 . The smallest value was $1.9 R_{S}$ in 2005 . Accordingly, the optimal source surface distances depict a rather large variation. Using the previously provided definition of cycle phases, the average optimal source surface distance is

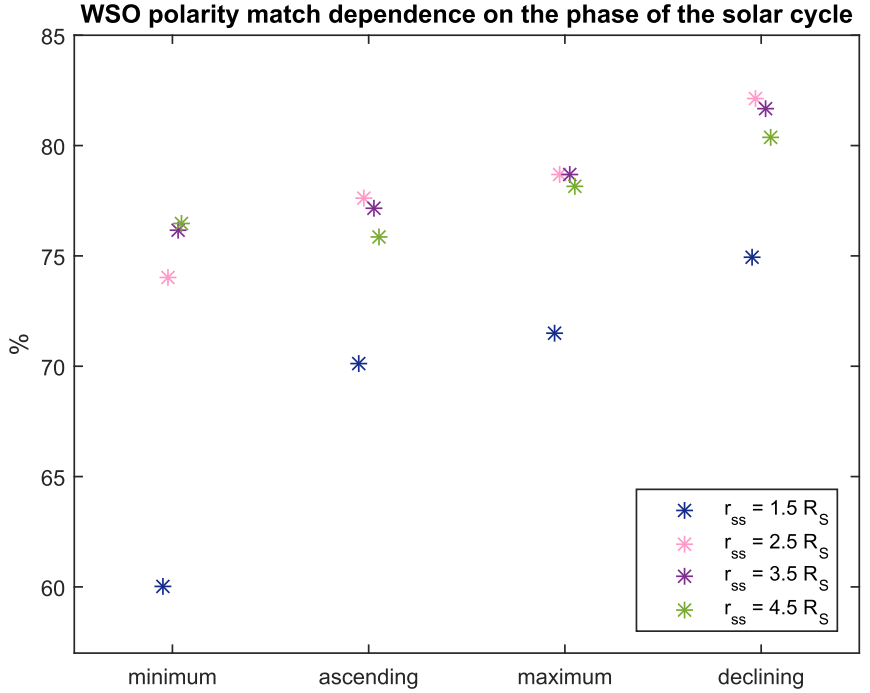

Figure 4. Average polarity match percentage for the four solar cycle phases over the whole WSO data period, using four source surface distances: $1.5 R_{S}$ (blue), $2.5 R_{S}$ (pink), $3.5 R_{S}$ (purple), and $4.5 R_{S}$ (green).

somewhat larger, about $3.3 R_{S}$, during solar minima than in the other three phases where the optimal source surface distances are roughly equal $\left(2.7 R_{S}\right.$ during maxima, $2.6 R_{S}$ during the declining phase, and $2.6 R_{S}$ during the ascending 
WSO optimal source surface
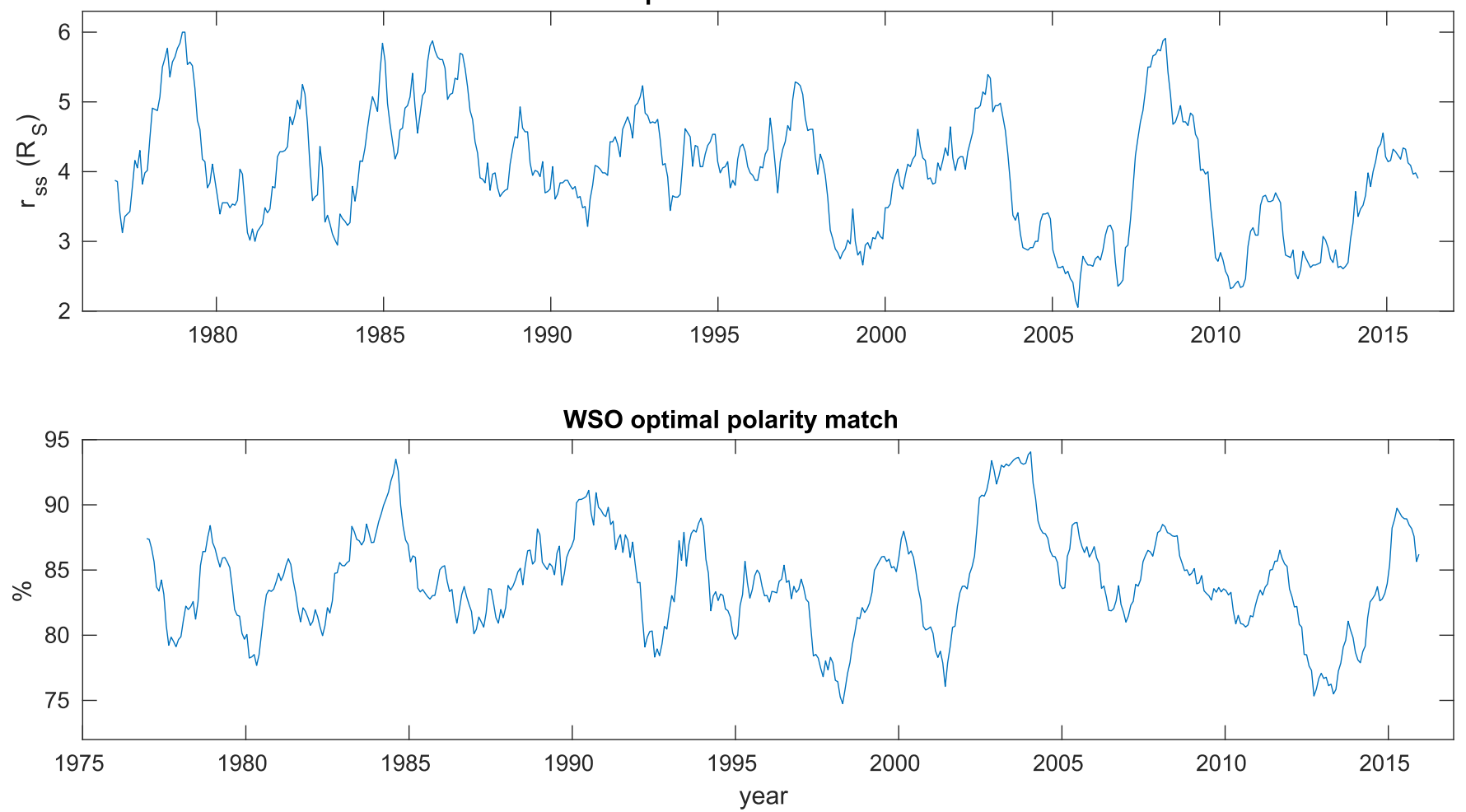

Figure 5. Top panel: the 13-rotation running means of the optimal source surface distances calculated for each Carrington rotation for WSO data. Bottom panel: the 13-rotation running means of the rotational polarity match percentages corresponding to the optimal source surface.

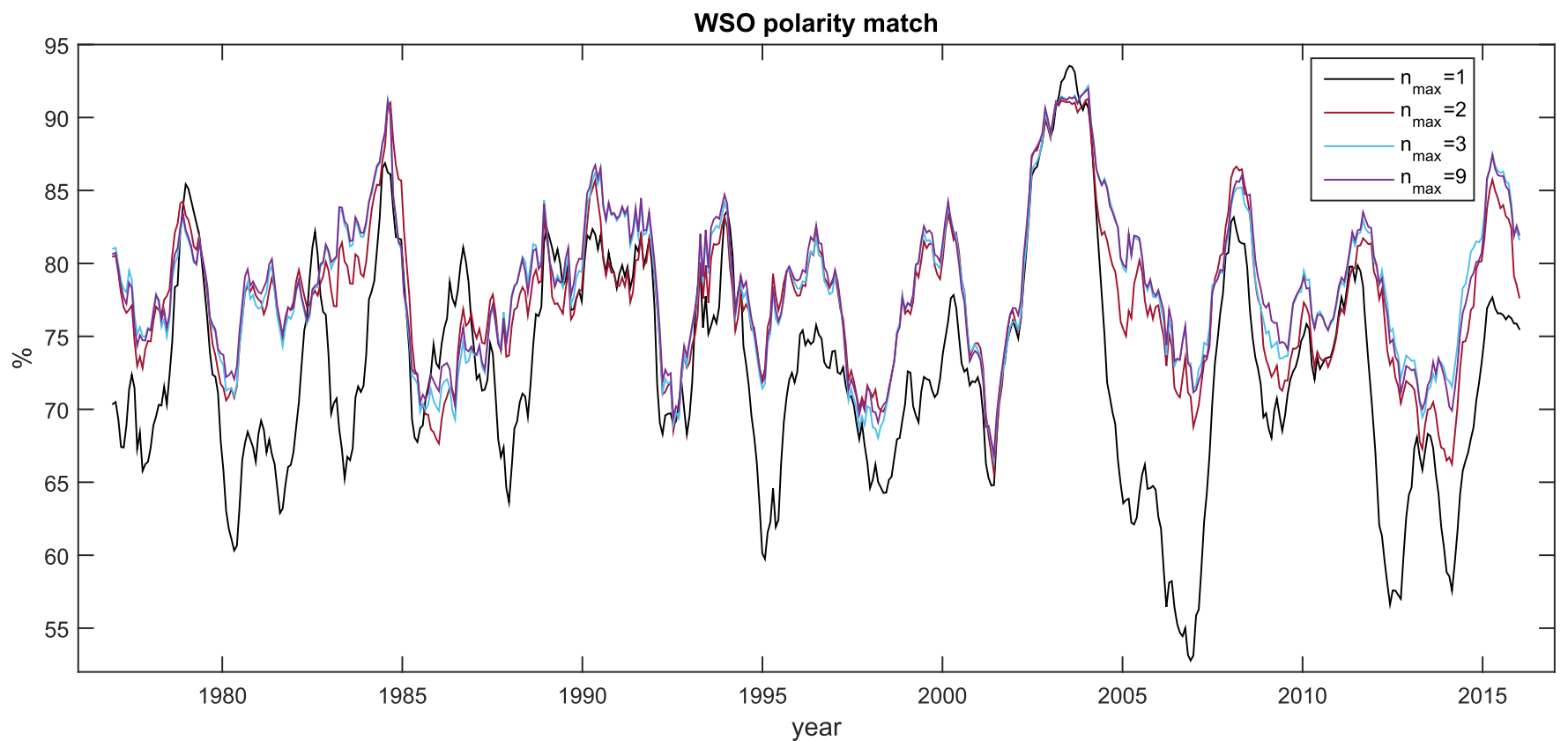

Figure 6. The 13-rotation running means of the WSO rotational polarity match percentage $\left(r_{s s}=3.5 R_{S}\right)$ for four different numbers of harmonic multipoles: $n_{\text {max }}=1$ (black), $n_{\max }=2$ (red), $n_{\max }=3$ (turquoise), and $n_{\max }=9$ (purple).

phase). Finally we note that, unless the later declining phase of the ongoing cycle 24 will reverse the trend, the average optimal source surface distance during cycle 24 will remain smaller than during all previous cycles. This reflects, most likely, the weaker activity and polar field strength during cycle 24.

The lower panel of Figure 5 shows the polarity match percentages corresponding to the optimal source surface distances. Note that the temporal variation of the optimal source surface polarity match percentage greatly resembles that of the three larger $r_{s s}$ values depicted in Figure 2, although the average level is, naturally, somewhat higher in Figure 5 (about 83\%) than in Figure 2 (about 78\%). Similar to Figure 2, the highest peaks of Figure 5 are found during the declining phase. 


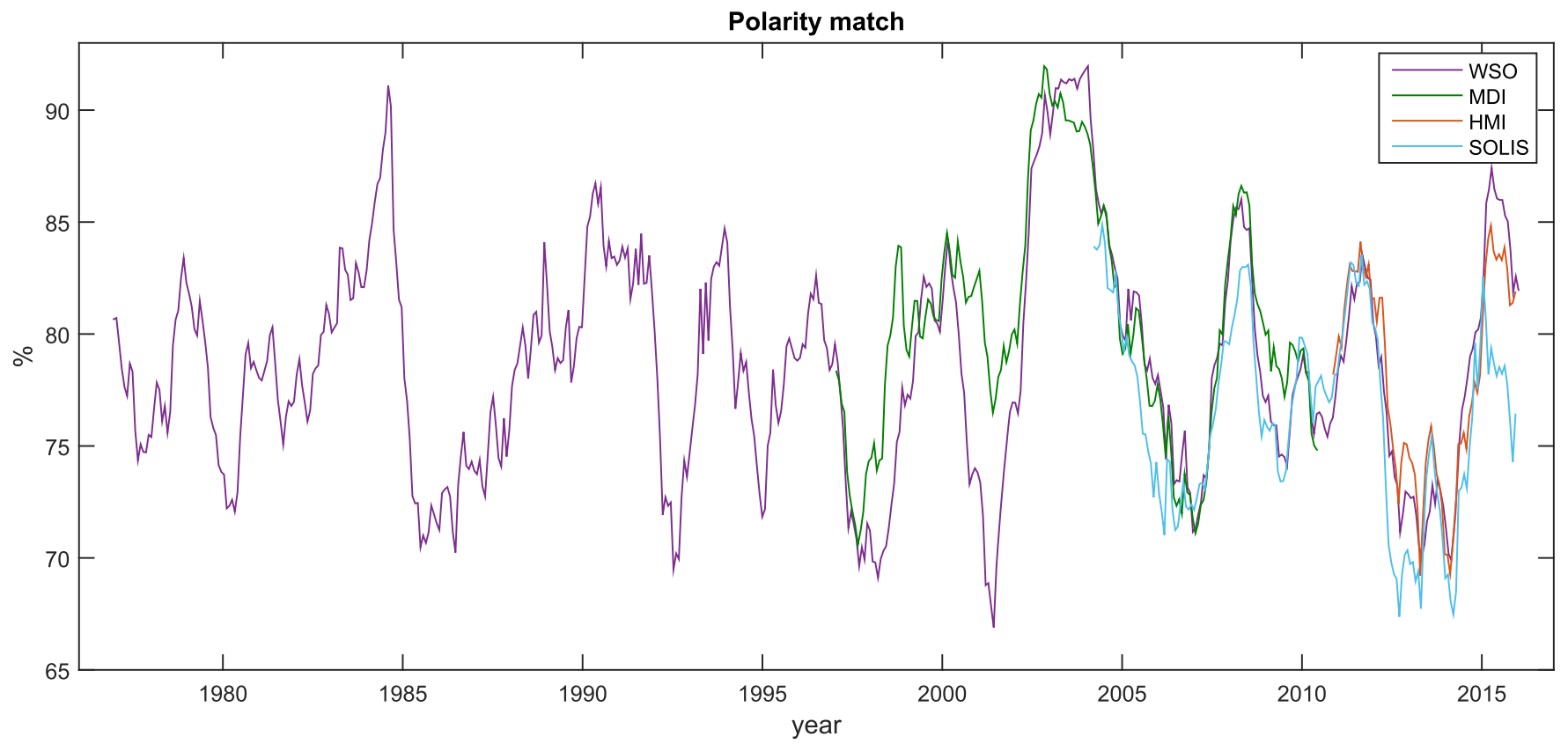

Figure 7. The 13-rotation running means of the rotational polarity match percentages $\left(r_{s s}=3.5 R_{S}, n_{\max }=9\right.$ ), calculated with photospheric data from WSO (purple), MDI (green), HMI (orange), and SOLIS (light blue).

\subsection{Dependence on Harmonic Order $n_{\max }$}

Another parameter that affects the polarity match is the number of multipoles, $n_{\max }$, in the spherical harmonic expansion of Equation (1). Figure 6 shows the polarity match calculated for four different values (1, 2, 3, and 9) of $n_{\max }$, with WSO data and $r_{s s}=3.5 R_{S}$. Having nine multipoles gives the best average match percentage $(78.55 \%)$, but the difference compared to three multipoles $(78.35 \%)$ or even to two multipoles (77.49\%) is very small. Only the dipole case $\left(n_{\max }=1\right.$, average polarity match of $\left.72.65 \%\right)$ is generally too coarse to give an equally good overall fit to the observed HMF polarity. However, note that there are certain periods (e.g., in 2001-2004) when the coronal field is very well approximated by the dipole term only. (Note that $n_{\max }=1$ includes the axial dipole $g_{10}$ and the two equatorial dipole terms $g_{11}$ and $h_{11}$, representing a tilted dipole field configuration). In fact, the highest (running mean) match percentage of $93 \%$ in the whole time series is found in 2003 using the dipole term only.

This comparison shows that the overall structure of the heliospheric magnetic field at 1 au is mostly rather simple and can be well described by only a few $\left(n_{\max }=2-3\right)$ harmonic terms. Note, however, that the match is never perfect, but cannot be improved by raising the longitudinal detail of the harmonic expansion. This suggests that the PFSS method is not capable of reproducing all details of the actual coronal field, or that synoptic maps lose some essential factors of the photospheric field, or that other factors exist (e.g., reconnection) that modify the heliospheric field during its pass from the corona to $1 \mathrm{au}$.

\subsection{Different Data Sets}

We have also examined the effect of using different data sets. Figure 7 shows the polarity match for WSO, MDI, HMI, and SOLIS for $r_{s s}=3.5 R_{S}$ and $n_{\max }=9$. Until 1996 there are data only from WSO. There is a very good correspondence in polarity match between the different data sets, except in 1998 and 2000-2002 when the WSO polarity match is significantly lower than MDI polarity match. Interestingly, a recent study (Virtanen \& Mursula 2016) found that WSO synoptic maps have problems (partly unknown earlier) exactly (and only) at these times. The good agreement between the four instruments at other times can be easily understood by a recent observation that the PFSS solutions based on the lowest harmonic coefficients are very similar in different data sets (I. Virtanen \& K. Mursula 2016, in preparation). Therefore the fact that $n_{\max }=3$ is sufficient to produce the large-scale structure of the coronal field explains why we get very similar polarity match percentages for these four data sets.

Between Carrington rotations 2008 and 2102 (from 2003 September to 2010 October), when there are simultaneous data from WSO, MDI, and SOLIS, the average polarity match percentages are, respectively, $79.3 \%, 79.3 \%$, and $77.8 \%$, showing that WSO and MDI yield an equally good average polarity match, while SOLIS performs slightly worse. For Carrington rotations from 2097 to 2177 (from 2010 May to 2016 June), when there are data from WSO, HMI, and SOLIS, the corresponding polarity matches are $77.6 \%, 77.6 \%$, and $75.5 \%$. Thus, WSO and HMI give a slightly better polarity match percentage than SOLIS.

Zhao et al. (2006) obtained a slightly higher success rate for predicting the HMF polarity with MDI (86.2\%) than with WSO $(84.5 \%)$ or Kitt Peak National Observatory $(85.5 \%)$ data during Carrington rotations 1911 and 2017 (from 1996 June to 2004 May). The polarity match we obtain for this period is $82.6 \%$ for MDI and $80.0 \%$ for WSO. The better match of MDI during this time is obviously due to the erroneous data in WSO during the previously mentioned years included in this interval.

We have also tested how the increasing number of multipoles affects the polarity match for SOLIS data, which has a considerably higher spatial resolution than WSO. The top panel of Figure 8 shows the SOLIS polarity match for four different $n_{\max }$ values. It shows that while $n_{\max }=1$ gives a significantly lower polarity match, there is only a very small difference 

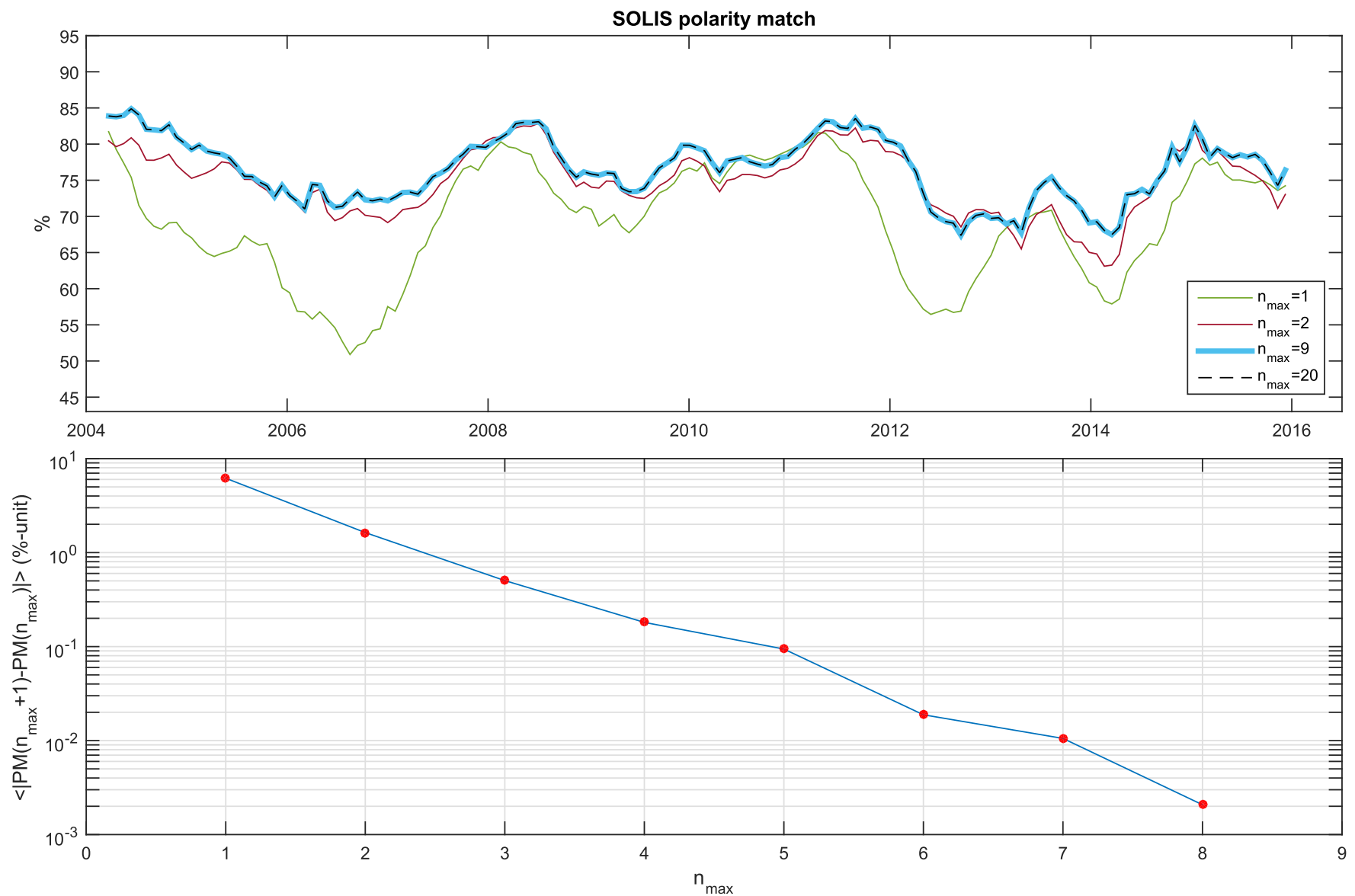

Figure 8. Top panel: the 13-rotation running means of the rotational polarity match percentages for SOLIS $\left(r_{s s}=3.5 R_{S}\right)$ for four different numbers of harmonic multipoles: $n_{\max }=1$ (green), $n_{\max }=2$ (red), $n_{\max }=9$ (blue), and $n_{\max }=9$ (black dashed line). Bottom panel: average absolute differences of rotational polarity matches for SOLIS between $\left(n_{\max }+1\right)$ and $n_{\max }$ for $n_{\max }=1-19$.

between $n_{\max }=2$ and $n_{\max }=9$, and between $n_{\max }=9$ and $n_{\max }=20$ there is no difference at all. To be more quantitative about this, we have calculated the absolute difference of rotational polarity matches between two consecutive $n_{\max }$ values, and then calculated the average over the whole SOLIS data period. The difference as a function of $n_{\max }$ is plotted in a logarithmic scale in the bottom panel of Figure 8 . Figure 8 shows that the decrease of the difference is roughly exponential. The difference between $n_{\max }=1$ and $n_{\max }=2$ is six percentage points, but between $n_{\max }=2$ and $n_{\max }=3$ it is only 1.6 percentage points, and between $n_{\max }=8$ and $n_{\max }=9$ it is only 0.002 . After $n_{\max }>9$ there is no difference in the polarity match.

\section{PREDICTING THE HMF RADIAL COMPONENT}

The decay of the radial magnetic field component follows a power law

$$
B_{r}(r)=B_{r}\left(r_{s s}\right)\left(\frac{r_{s s}}{r}\right)^{p},
$$

where according to Maxwell's equations, the value of $p$ should be 2 . This provides a simple tool to evaluate the accuracy of the strength of the HMF predicted by the PFSS model. Solving $p$ from the previous equation gives

$$
p=\frac{\ln \left|B_{r}(1 \mathrm{au}) / B_{r}(s s)\right|}{\ln \left(r_{s s} / 1 \mathrm{au}\right)}
$$

We have calculated the average of $p$ for each rotation. The 13rotation running means of the rotational averages of $p$ are plotted in Figure 9 for four source surface distances from $1.5 R_{S}$ to $4.5 R_{S}$.

The smallest source surface distance of $1.5 R_{S}$ gives the highest values of $p$, and the $p$-values decrease systematically with source surface distance. The average $p$-value over the whole data period is 1.73 for the source surface distance of $1.5 R_{S}, 1.51$ for $2.5 R_{S}, 1.37$ for $3.5 R_{S}$, and 1.26 for $4.5 R_{S}$. This dependence of $p$ on source surface distance is expected since, as explained previously, a small source surface confines less of a coronal field inside the source surface, yielding a stronger open solar flux and a larger $p$-value to match with the HMF field at 1 au.

The power $p$ varies fairly systematically and quite strongly over the solar cycle, being highest at and soon after sunspot maxima and lowest during minima. A fairly similar cyclic variation is found for all four source surface distances. The solar cycle variation in $p$ is caused by the structural changes in the coronal magnetic field and, in particular, by the mean location of the heliospheric current sheet (HCS) over the course of the solar cycle, as explained by Mursula \& Virtanen (2011). During solar minimum the HCS is flat and the Earth is long located close to the HCS, so the source latitude of the observed HMF is close to the neutral line on the source surface. Since the PFSS field value is (excessively) weak close to the neutral line (see also the "Discussion" section), $p$ becomes smaller at these times. 


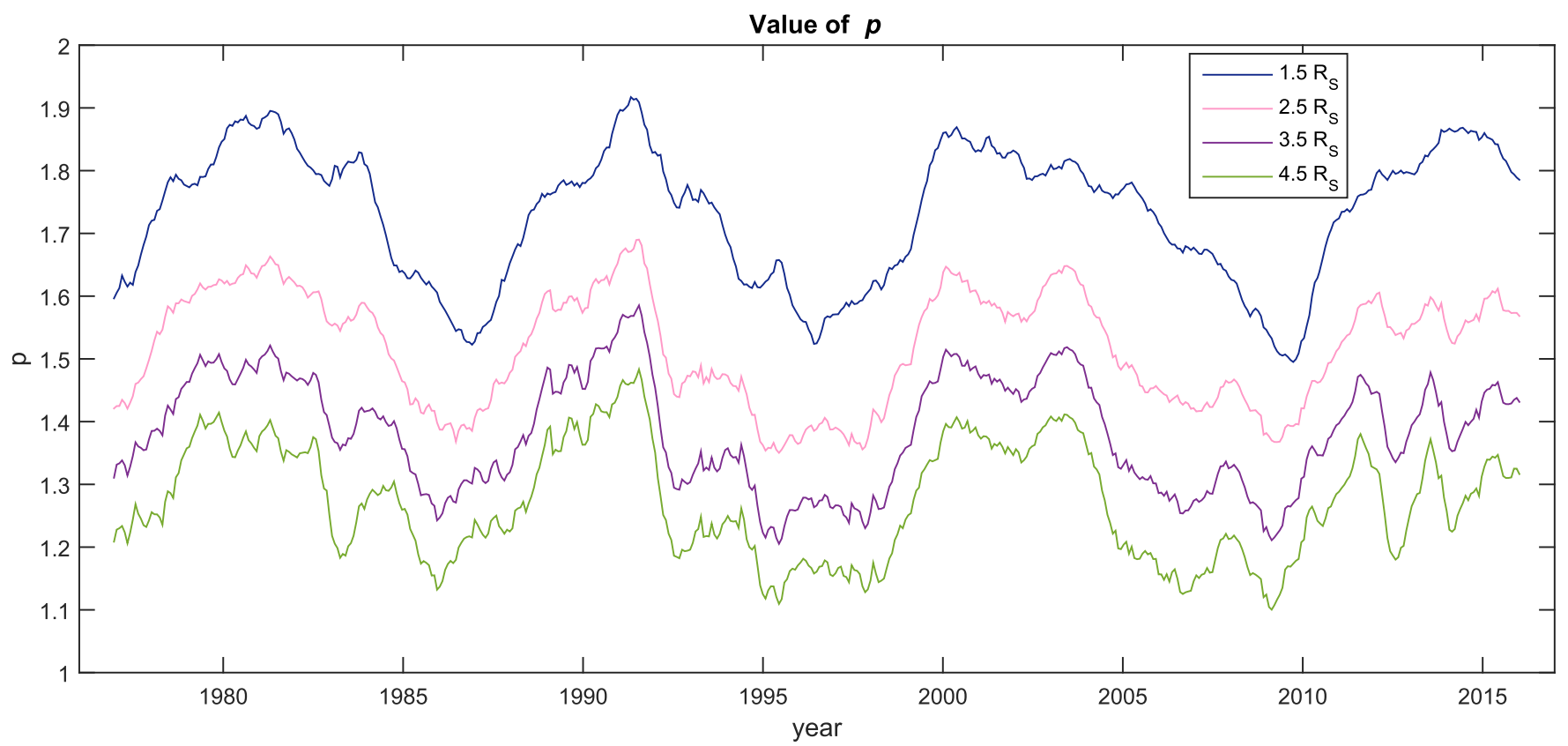

Figure 9. The 13-rotation runnings mean of the rotational averages of $p$ (WSO, $n_{\max }=9$ ) for four source surface distances: $1.5 R_{S}$ (blue), $2.5 R_{S}$ (pink), $3.5 R_{S}$ (purple), and $4.5 R_{S}$ (green).

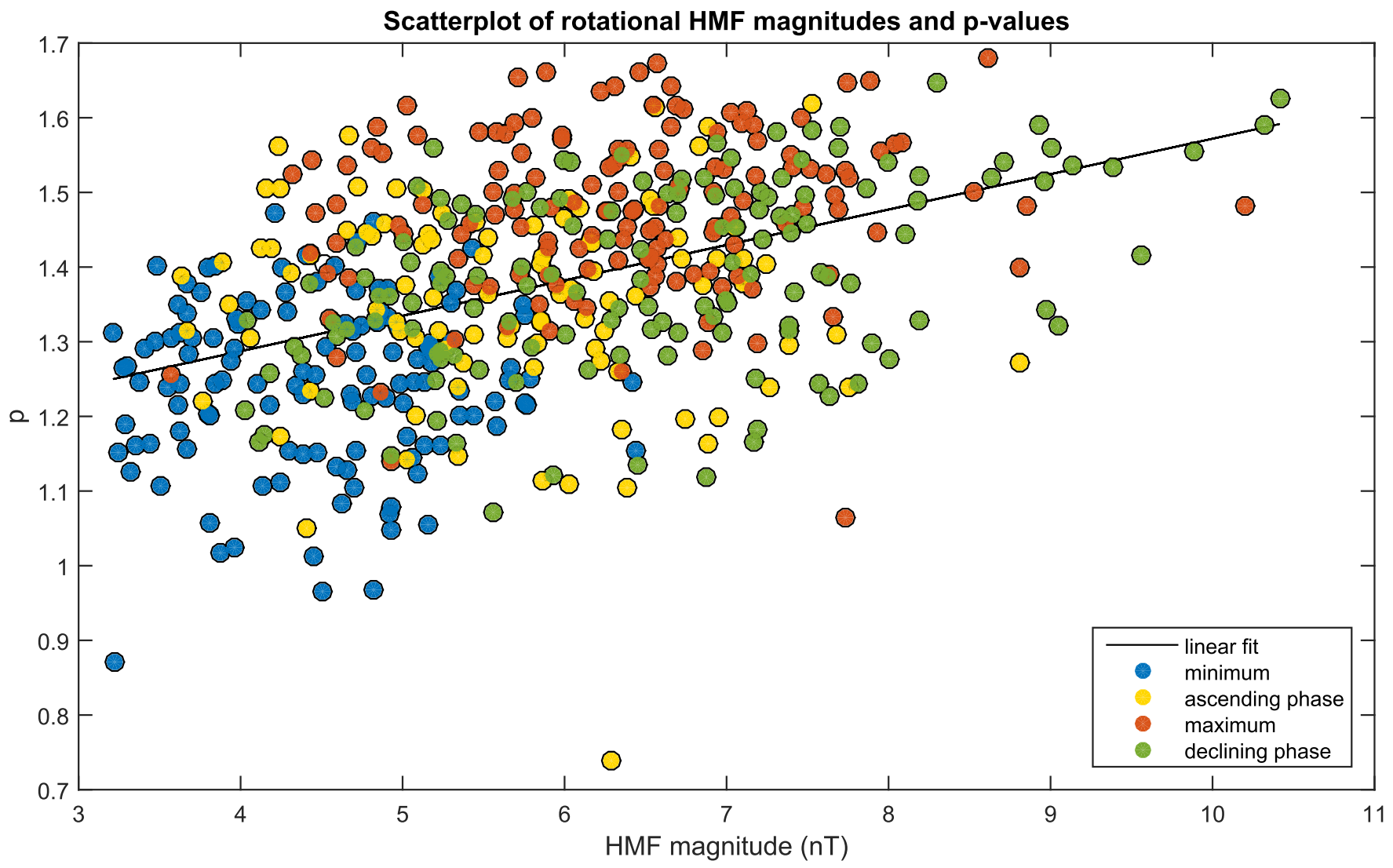

Figure 10. Scatter plot of rotational averages of $p\left(r_{s s}=3.5 R_{S}, n_{\max }=9\right)$ and the absolute magnitudes (B) of the HMF at 1 au. Blue filled circles denote the minimum, yellow denotes the ascending phase, red denotes the maximum, and green denotes the declining phase. Black lines show the linear fit to all data points.

Figure 10 shows a scatter plot of the rotational $p$-values and the absolute values of the HMF at 1 au. There is a significant positive correlation $(\mathrm{cc}=0.4440, p<0.0001)$, mainly driven by large field values of the maximum and declining phase. All data points during minimum show a rather low HMF value, whereas the high values occurred mainly during the maximum or the declining phase, reflecting the well known solar cycle variation of the HMF (Wang \& Sheeley 2002; Zhou \& Smith 2009).

Figure 11 compares the $p$-values for the four different data sets for $r_{s s}=3.5 R_{S}$ and $n_{\max }=9$. A very similar long-term (solar cycle) evolution in $p$ is seen for all four data sets. 


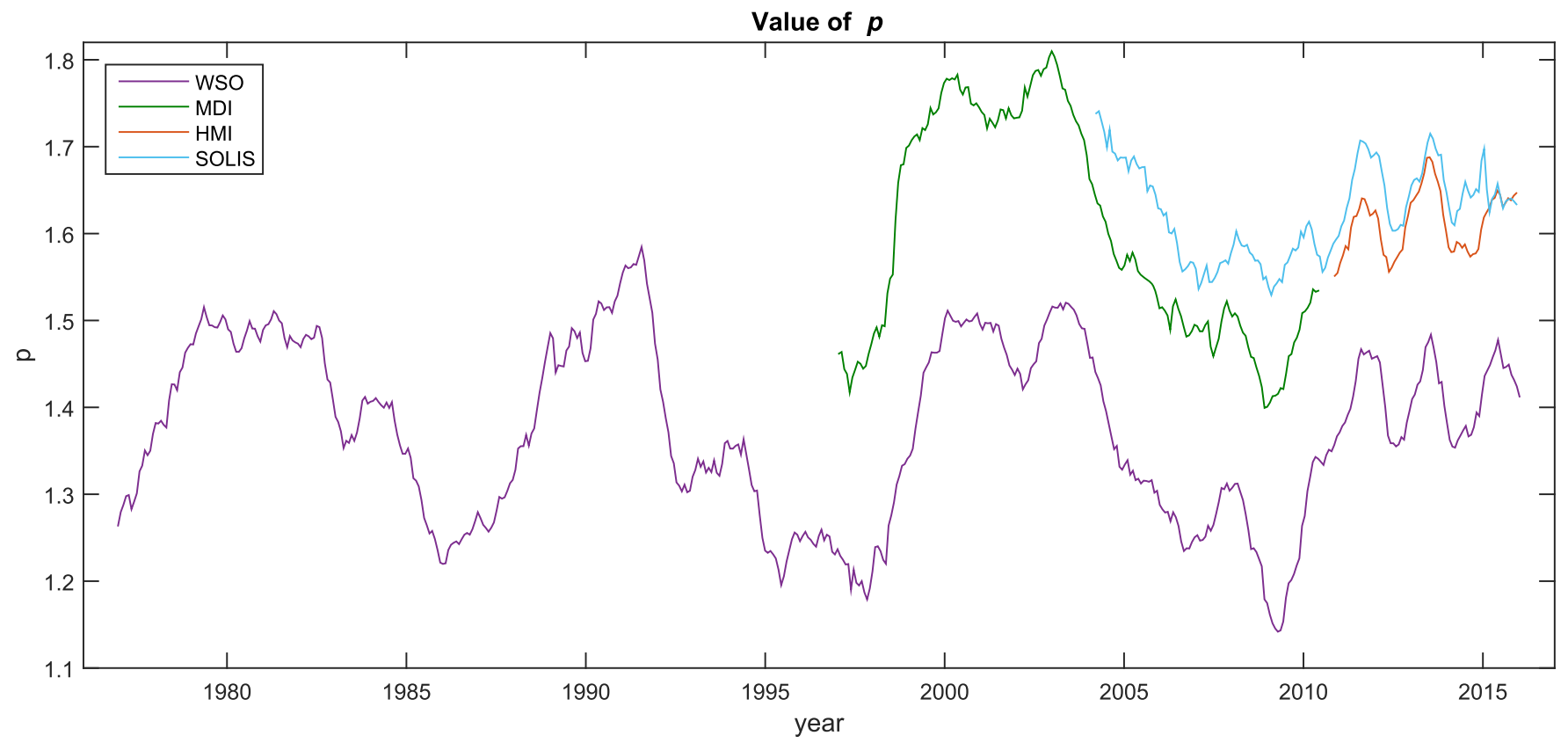

Figure 11. The 13-rotation running means of $p$ using WSO (purple), MDI (green), HMI (orange), and SOLIS (blue) data $\left(r_{s s}=3.5 R_{S}, n_{\max }=9\right.$ ).

However, Figure 11 clearly shows that WSO yields a systematically lower $p$-value than the others. The average value of $p\left(r_{s s}=3.5 R_{S}, n_{\max }=9\right)$ for WSO over its full data period is 1.37. For MDI, HMI, and SOLIS, the average of $p$ over their full data periods is $1.60,1.61$, and 1.63. SOLIS also yields slightly larger $p$-values than MDI and HMI during the overlapping time. The effect of saturation during field observation is strongest in WSO, which causes the intensity level of WSO synoptic maps to be lower than for the other magnetograms (Riley et al. 2014; Virtanen \& Mursula 2016). Saturation may occur if the magnetic field of the active region is very high; hence the Zeeman shifted spectral line will be outside of the spectrometer slit and cannot be detected. Moreover, with a low resolution, the size of one pixel may be significantly larger than the size of an active region, in which case most of the contribution to the spectral line comes from the low intensity magnetic field, called the filling factor. This makes the spectral line of the active region appear only at the wing of the spectral line of the filling factor, making it impossible to measure.

\section{DISCUSSION}

\subsection{Polarity Match: Long-term Evolution}

The temporal variability of the match percentage depicted in, for example, Figure 7 can be explained by the changes in the structure of the solar magnetic field and in particular of the HCS during the solar cycle. Figure 12 shows the WSO tilt angles of the HCS in corona, and Figure 13 shows the histogram plot for the yearly number of sector crossings detected at 1 au in OMNI 2 data, normalized by the OMNI 2 data coverage percentage. The number of observed sector crossings is obviously linked to the structure of the HCS, in particular its tilt. For example, during the declining phase, the number of sector crossings is relatively low, because of the dominant two-sector structure of the HMF, due to a tilted and fairly thin HCS. On the other hand, during solar minimum the number of sector crossings is large because the HCS is rather untilted, and the Earth repeatedly moves across the nearby HCS from one sector to the other.

Figure 7 (and other similar figures) shows low values of match percentages (most often local minima) during all solar minima, starting in 1977. After 1977, the polarity match increases during the ascending phase, when the HCS is becoming more tilted. There is a minimum in match in 1980, when the field configuration gets more complicated during the solar maximum. Figure 12 shows a tilt maximum and Figure 13 a high number of sector crossings at this time. The polarity match rises, thereafter, during the declining phase until 1984, in a good agreement with the decreasing tilt (Figure 12) and a decreasing number of sector crossings (Figure 13). During the declining phase, polar coronal holes extend toward the equator, making the HMF sector structure better defined. Surprisingly, the match percentage decreases dramatically from 1984 to 1985. Interestingly, this is exactly the time when the HCS starts being systematically shifted southward for about 3 years, marking the so-called bashful ballerina period (Mursula \& Hiltula 2003; Zhao et al. 2005; Virtanen \& Mursula 2016). (We will study the effect of the HCS shift in more detail in a separate paper; J. S. Koskela et al. 2016, in preparation.) The number of sector crossings is also large in 1985-1988. The polarity match starts to rise again after the minimum during the ascending phase, when the HCS activates, reaching a maximum in 1990. This development is coincided by the reduction of number of sectors from 1986 to 1990 (Figure 13). The match percentage is only weakly reduced in 1989, at the time of sunspot maximum, showing that the field structure may be rather simple even during sunspot maxima.

The development of the match percentage during the declining phase of cycle 22 is fairly complicated. Another peak in the percentage is found in 1994, simultaneous with a minimum in the number of sectors (Figure 13). However, this peak is surrounded by deep minima in 1992 and in 1995. (Later, there is another minimum around the sunspot minimum in 1996-1997). Also, the number of sector crossings is quite high in 1991 and 1992, which implies that the sector structure 


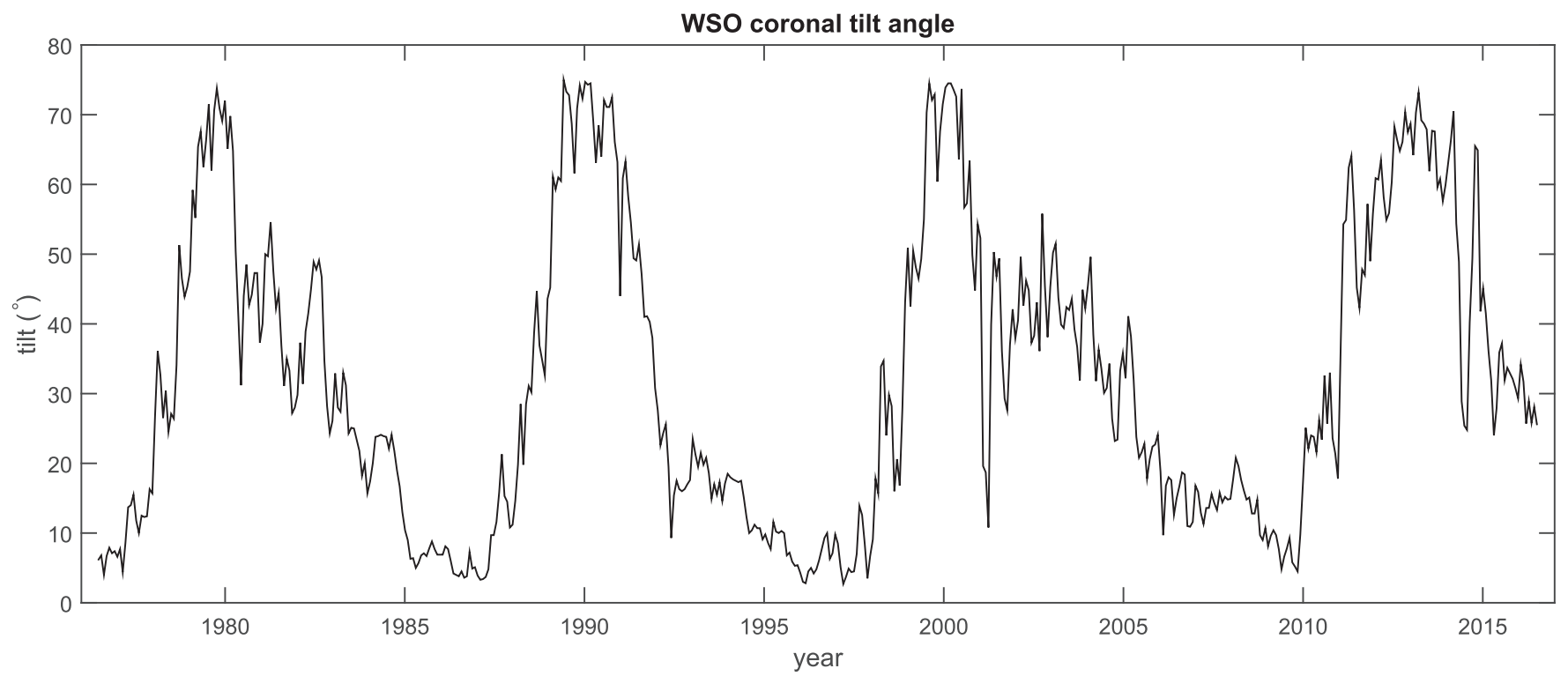

Figure 12. Rotational tilt angles of the HCS in corona from WSO $\left(r_{s s}=3.25 R_{S}\right)$. Data are available at http://wso.stanford.edu/Tilts.html.

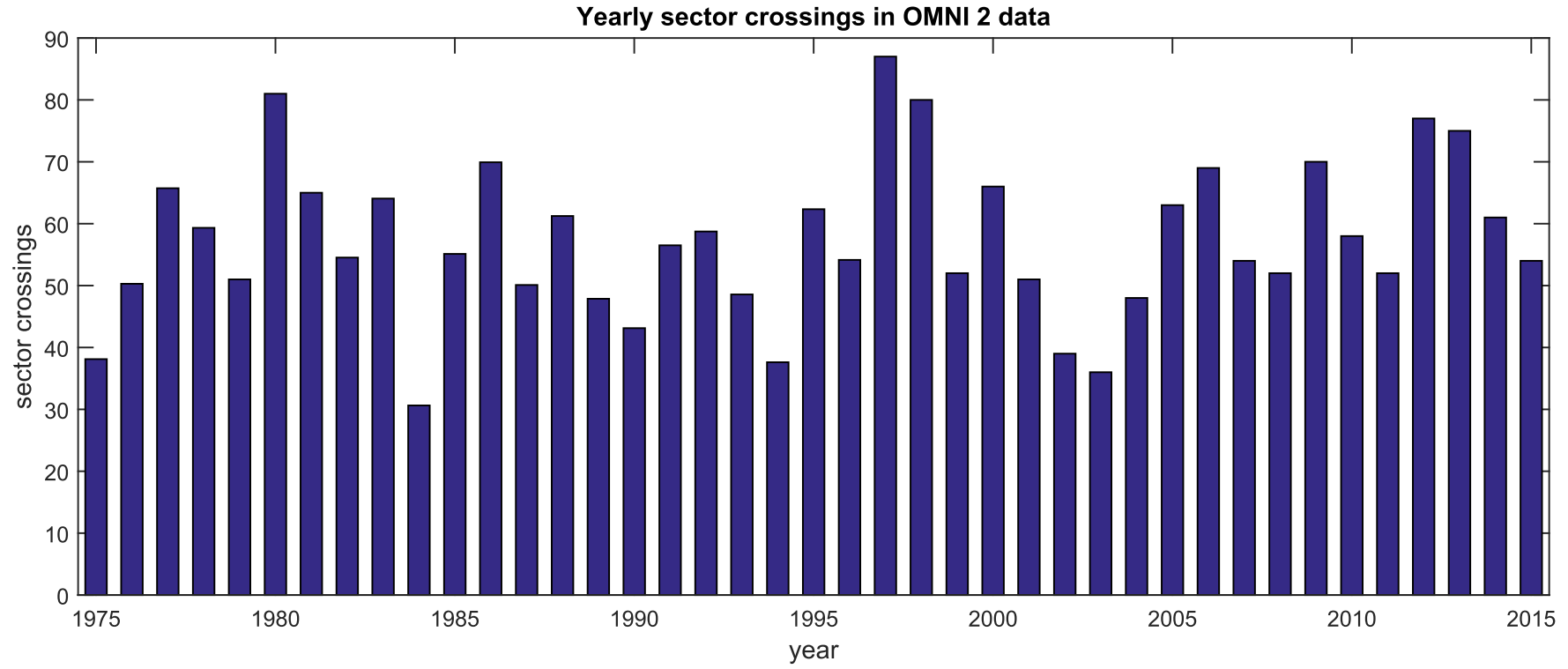

Figure 13. Number of yearly sector crossings at 1 au according to OMNI 2 data, normalized by data coverage. The sectors are defined according to plane division.

was complicated. Figure 12 shows that the tilt of the HCS decreased very rapidly and quite early in the declining phase of cycle 22 . We also note that the HCS was southward shifted in 1992-1995 (Virtanen \& Mursula 2016), in agreement with decreasing match since 1992. However, the peak in 1994 is most likely related to the activation of the southern polar coronal holes at this time, as described by Mursula \& Virtanen (2012).

After the minimum in 1997 the polarity match percentage rise again in the ascending phase, making a shallow (according to MDI) minimum at the sunspot maximum in 2001. The declining phase in 2002-2003 shows the highest polarity match percentages in the whole data series. In 2002-2003 the number of sector crossings was also very low; 2003 marks the lowest after 1984 (Figure 13). During this declining phase the HCS tilt angle was quite high $\left(40^{\circ}-50^{\circ}\right)$, contrary to the previous cycle (Figure 12). There is another surprising drop in the match percentage in 2005-2006, which coincides with a small tilt angle $\left(15^{\circ}\right)$ and an increasing number of sector crossings. Similarly to the previous solar cycle there is another peak in polarity match percentage in the late declining phase, before the polarity match minimum of solar minimum in 2008-2009. Also, similar to that described earlier, these changes in polarity match are related to the HCS asymmetry, although the asymmetry in cycle 23 was overall less systematic at 1 au than in earlier cycles (Mursula \& Virtanen 2011).

After the minimum in 2009 , the match percentage again increases during the ascending phase of cycle 24 . However, the reduction of a polarity match around the maximum of cycle 24 in 2012 and 2014 is exceptionally deep and long. Finally, during the early declining phase, the polarity match starts rising again.

\subsection{Polarity Match: Number of Harmonics}

Figure 6 shows that there is one long interval prior and during the minimum in 1985-1986 (and two shorter intervals at 
other times) when the dipole $\left(n_{\max }=1\right)$ gives a clearly better polarity match than using a larger number of multipoles. Thus, during this minimum, the field at 1 au can be best approximated by a tilted dipole. However, the same situation is not valid during the other three sunspot minima, 1976-1977, 1996-1997, and 2009, when the $n_{\max }=1$ case gives somewhat smaller match percentages than the higher $n_{\max }$ cases. This emphasizes the uniqueness of the solar minimum in the mid1980s. This situation is most likely related to the fact (Richardson \& Paularena 1997) that the HCS was exceptionally thin during this minimum, reflecting exceptionally strong polar fields. As during all minima, there were several sector crossings during these years (see Figure 13), reflecting the flatness of the HCS and the Earth's proximity to the HCS. However, because of the exceptionally thin HCS, the HMF sectors were more clearly organized than during other minima, leading to a better polarity match.

The pure dipole field gives at least a slightly weaker match than higher $n_{\max }$ fields during most times in all ascending phases and all maxima. During the ascending phase, the dipole field is weakening and the higher multipoles start playing an increasing role. This is particularly clear during the last two ascending phases in 1998-1999 and in 2011-2012, and during the maximum in 1980, 2000, and in particular, during the last maximum in 2014 (see Figure 6). The large difference between the dipole and higher $n_{\max }$ cases since the maximum of cycle 23 is most likely related to the changes in sunspots and other magnetic elements observed at this time (Svalgaard \& Hudson 2010; Lukianova \& Mursula 2011; Clette \& Lefèvre 2012).

Figure 8 shows that the polarity match percentages very rapidly approach to a limit value when increasing $n_{\max }$. In fact, the mean absolute difference in match percentage for SOLIS data (see Figure 8 ) between $n_{\max }=2$ and $n_{\max }=3$ is only 1.6 percentage points. Also, the limit value of match percentages is already reached for $n_{\max }=9$, beyond which the coronal structure remains exactly the same. This dependence is partly due to the effective reduction of the higher harmonic terms in the coronal field by the powers of the ratio of the source surface and photospheric distances, partly by the implemented longitudinal smoothing (see Section 2), whereafter the longitudinal accuracy was reduced to $13^{\circ} .7$.

Independently, the choices made by Zhao et al. (2006) also led to the fact that they did not see any change in the field after increasing the number of multipoles beyond nine with MDI data.

\subsection{Polarity Match: Source Surface Distance}

The lowest source surface distance of $1.5 R_{S}$ gives a significantly lower match, especially during all minima and ascending phases (see Figure 2). The two largest source surface distances of $3.5 R_{S}$ and $4.5 R_{S}$ give a better match percentage than $2.5 R_{S}$ or $1.5 R_{S}$, especially during the minimum in 1986-1987 (and less in 1995-1997). This is most likely related to the previously mentioned fact that the (single) dipole term matches best the field during this minimum (due to a record thin HCS). For a larger source surface distance, the higher harmonics are more strongly reduced, making the field effectively more dipolar. On the other hand, the source surface distance of $2.5 R_{S}$ gives the best match during the declining phase in 2005-2006.

Figure 5 shows the long-term variation of the optimal source surface distance. During solar minima, the low latitudes are dominated by large-scale coronal loops, which tends to raise the height at which the field becomes radial (i.e., the source surface distance). On the other hand, the coronal field during solar maxima consists of several smaller scale loops and small regions of open field intermingled, leading to a lower source surface distance at this time. On average the optimal source surface distance during solar maxima is about $2.7 R_{S}$, and during solar minima it is about $3.3 R_{S}$ (i.e., about $25 \%$ larger than during maxima).

\subsection{Radial Field Component}

The power $p$ (see Figure 9) follows the solar cycle variation of the structure and intensity of the HMF. In particular, the flatness of the HCS and the proximity of the HMF field source to the HCS produce the minima of $p$ during sunspot minima (Mursula \& Virtanen 2011). The PFSS model gives low field values close to the neutral line because the field goes continuously to zero before changing its sign (Zhao \& Hoeksema 1995). This latitude dependence of the radial component of the magnetic field is an artifact of the PFSS model.

The WSO data set gives a lower value for $p$ (and thus a weaker coronal field strength) than the other data sets (see Figure 11). Scaling factors are needed when comparing synoptic maps from different observatories (Riley et al. 2014; Virtanen \& Mursula 2016). Our results show that determining the value for $p$ is dependent on both the data source and the chosen source surface distance. Accordingly, the total (unsigned) coronal flux also depends on them.

Lee et al. (2011) calculated the total unsigned open solar flux using MWO and MDI photospheric data and the PFSS model with different source surface distances, and compared it with the open solar flux measured at 1 au $\left(F=4 \pi(1 a u)^{2}\left|B_{r}\right|\right)$. Based on this comparison, they obtained surprisingly small optimal source surface values for the two solar minima in 1996 and $2009\left(1.8-1.9 R_{S}\right.$ for MWO and $1.5 R_{S}$ for MDI). Arden et al. (2014) compared the total unsigned open solar flux at 1 au and in corona in 1996-2012 using photospheric data from MDI and HMI. They concluded that the optimal source surface for the maximum of solar cycle 23 is $2.5 R_{S}$, and it increases toward the declining phase of the cycle. During the solar minimum in 2007-2008 they found the average optimal source surface of $3.28 R_{S}$. The optimal source surface distances found by Arden et al. (2014) for solar maxima and minima are in a very good agreement with those found here. On the other hand, the optimal source surface found here is significantly larger than that by Lee et al. (2011).

We note that there is a problem with determining the optimal source surface distance using the total open solar flux, as done by both Lee et al. (2011) and Arden et al. (2014). In this method the flux calculated at the source surface is dependent not only on the source surface distance, but also on the scaling of the magnetograms: if the magnetogram scaling factor is too small (large), also the source surface needs to be decreased (increased) in order to match the open solar flux value at $1 \mathrm{au}$. On the other hand, the polarity comparison used here is not affected by the magnetogram scaling and, as we have shown, not significantly on the number of multipoles, either. The only parameter that affects the polarity match is the source surface distance.

The open flux comparison is related to our $p$-value comparison. The difference is that the total open solar flux is 
integrated over the whole source surface, whereas we do a ballistic comparison. While the calculation of the total open solar flux is not affected by the proximity to the HCS, the $p$ values are, due to the previously mentioned artifact of the PFSS model. Comparisons are also affected by the different data sets used: the absolute level of MDI and HMI synoptic map fields is higher than that of WSO.

\section{SUMMARY}

We have used the PFSS model to calculate the coronal magnetic field and to compare it with the heliospheric field measured at $1 \mathrm{au}$. The polarity prediction is best, on average, during the declining phase of the solar cycle $(82 \%)$ because of the thin and tilted HCS, which gives a clear, mostly two-sector structure in the heliosphere. During the maxima the average polarity match is $78 \%-79 \%$, during the ascending phase it is $76 \%-78 \%$, and during the minima it is $74 \%-76 \%$. We have calculated the polarity match and power of radial decay, $p$, of the radial field for WSO from 1976 to 2016, for MDI from 1996 to 2011, for HMI from 2010 to 2016, and for SOLIS from 2004 to 2016.

We have tested the effect of changing the model variables (source surface distance $r_{s s}$ and the number of multipoles $n_{\max }$ ) on polarity match. We found the best polarity match for the source surface distance is $3.25 R_{S}(78.6 \%)$. However, the $r_{s s}$ values between $2.25 R_{S}$ and $5.75 R_{S}$ yielded matches within two percentage points from the maximum. An average optimal surface distance of about $2.7 R_{S}$ was obtained for solar maxima, whereas during solar minima the optimal source surface is higher, at about $3.3 R_{S}$. The overall polarity match does not much improve when the number of harmonic terms is raised beyond $n_{\max }=2$. However, there are a few time periods, the longest in 1985-1986, when the dipole $\left(n_{\max }=1\right)$ alone best describes the coronal field due to an exceptionally thin HCS. All four studied data sets (WSO, MDI, HMI, and SOLIS) give a very similar time evolution and a fairly similar absolute level of polarity match predictions.

While all four data sets agree on the time evolution of $p$, WSO gives a significantly lower value for $p$ than the other three data sets. This shows that determining the value for $p$ is dependent not only on the source surface distance, but also on the data source. This complicates the comparisons of coronal field intensity obtained from different data sets, making the results on coronal fields and source surface distances data set dependent. On the other hand, results of optimal source surface distance based on polarity match, as done here, are more universal.

We acknowledge the financial support by the Academy of Finland to the ReSoLVE Centre of Excellence (project No. 272157). Wilcox Solar Observatory data used in this study were obtained via the website http://wso.stanford.edu, courtesy of J.T. Hoeksema. Data were acquired by SOLIS instruments operated by NISP/NSO/AURA/NSF. SOHO/ MDI is a project of international cooperation between ESA and NASA. HMI data are courtesy of the Joint Science Operations Center (JSOC) Science Data Processing Team at Stanford University. The OMNI data were obtained from the GSFC/ SPDF OMNIWeb interface at http://omniweb.gsfc.nasa.gov.

\section{REFERENCES}

Altschuler, M. D., \& Newkirk, G. 1969, SoPh, 9, 131

Arden, W. M., Norton, A. A., \& Sun, X. 2014, JGRA, 119, 1476

Clette, F., \& Lefèvre, L. 2012, JSWSC, 2, A06

Hoeksema, J. T., Wilcox, J. M., \& Scherrer, P. H. 1983, JGR, 88, 9910

Lee, C. O., Luhmann, J. G., Hoeksema, J. T., et al. 2011, SoPh, 269, 367

Lockwood, M., Rouillard, A. P., Finch, I., \& Stamper, R. 2006, JGRA, 111, A09109

Lukianova, R., \& Mursula, K. 2011, JASTP, 73, 235

Maliniemi, V., Asikainen, T., \& Mursula, K. 2014, JGRD, 119, 9752

Mursula, K., \& Hiltula, T. 2003, GeoRL, 30, 2135

Mursula, K., \& Virtanen, I. 2011, A\&A, 525, L12

Mursula, K., \& Virtanen, I. I. 2012, JGRA, 117, A08104

Ness, N. F., \& Wilcox, J. M. 1964, PhRvL, 13, 461

Parker, E. N. 1958, ApJ, 128, 664

Richardson, I. G., \& Cane, H. V. 2012, JSWSC, 2, A02

Richardson, J. D., \& Paularena, K. I. 1997, GeoRL, 24, 1435

Riley, P., Ben-Nun, M., Linker, J. A., et al. 2014, SoPh, 289, 769

Schatten, K. H., Wilcox, J. M., \& Ness, N. F. 1969, SoPh, 6, 442

Smith, E. J., \& Balogh, A. 1995, GeoRL, 22, 3317

Sun, X. 2009, Notes on PFSS Extrapolation, http://wso.stanford.edu/words/ pfss.pdf

Svalgaard, L., \& Hudson, H. S. 2010, in ASP Conf. Ser. 428, SOHO-23: Understanding a Peculiar Solar Minimum, ed. S. R. Cranmer, J. T. Hoeksema, \& J. L. Kohl (San Francisco, CA: Astronomical Society of the Pacific), 325

Virtanen, I., \& Mursula, K. 2016, A\&A, 591, A78

Virtanen, I. I., \& Mursula, K. 2010, JGRA, 115, A09110

Wang, Y.-M., \& Sheeley, N. R. 2002, JGRA, 107, 1302

Wiegelmann, T., Petrie, G. J. D., \& Riley, P. 2015, SSRv, 75

Zhao, X., \& Hoeksema, J. T. 1995, JGR, 100, 19

Zhao, X. P., Hoeksema, J. T., Liu, Y., \& Scherrer, P. H. 2006, JGRA, 111, A 10108

Zhao, X. P., Hoeksema, J. T., \& Scherrer, P. H. 2005, JGRA, 110, A10101

Zhou, X., \& Smith, E. J. 2009, JGRA, 114, A03106 